\title{
Study on the Idealist and Realistic Style in the Songs of Chu a Case Study of Qu Yuan 's Works
}

\author{
Shiqian Deng \\ Sichuan Agricultural University, Ya’an, Sichuan, 625000
}

Keywords: Natural Geographical Environment, Social Environment, Mythology and Religion

\begin{abstract}
The Songs of Chu refer to "poems created with the local music, language and name of Chu." There is a clear distinction between the form and the northern poems, but the difference is not only reflected in the construction of the poetic style, the two language styles, ideological rationale, and even the value orientation has a more obvious difference. In contrast, the Songs of Chu in the structure has a longer sentence and length, in the language there are more obvious modification, in the content of a more magnificent imagination, more elegant in the emotional and elegant, in the ideological More emphasis on the absolute spirit of advocating ...... Therefore, it is easy to see the idealist of The Songs of Chu and the resulting idealist style, on this point of view, we will elaborate in this article, and we also proposed the new proposition of "the realism of the speech", and discusses the mutual integration of the interaction between the two and their influence on the style, style and even value orientation of later literature.
\end{abstract}

\section{Introduction}

"Chu" is the original meaning of Chu with the characteristics of the text, or, more broadly speaking, is Chu's folk songs, since it is a folk song, then with Chu features also take for granted. But also the folk songs, The Songs of Chu and the northern folk songs, that is, later collected and compiled into the "Book of Songs", but also has a distinct difference. Simply put, the two in the structure, emotional expression and ideological connotation and other internal and external construction are different. More obvious in the structural form and creative style. And in terms of the more essential part of the creative style is the most fundamental difference between the two direct reflection. It is well known that "the Book of Songs" is more realistic style, and that The Songs of Chu is more idealistic. "In this paper, we have to propose a new proposition that both the idealist and the realist The

Before talking about the style of creation, I think it is necessary to clarify the concept of The Songs of Chu again. The original meaning of "the original meaning of The Songs of Chu refers to the distinctive features of the rhetoric", which is the original meaning of the Songs of the Songs, but also the general meaning of The Songs of Chu, and once involved in the "creative style" of the topic, then The Songs of Chu in the production and life of spontaneous creation of folk songs, I am afraid that properly. Therefore, we may wish to use another saying that "The Songs of Chu is the poem with the local tones, language and name creation", the core of this definition lies in the "poem", that poet independent creation, that is, the writers of the works. Based on this understanding, it is more appropriate to talk about the style of creation.

\section{The Formation and Expression of Idealist Style}

As an important representative of Qu Yuan, "Li Sao" has a clear idealistic style. "Li Sao" Although it is based on Qu Yuan's own life and make, but there is no lack of a lot of idealistic description and expression. Here the "idealism" has two aspects of connotation, on the one hand refers to the reality of the magnificent magical imagination, on the other hand refers to the poet for the reality of the struggle is also idealized.

In "Lament", the magnificent imagination is almost everywhere, is that is. The most obvious is 
that the numerous forest of the total of the grass, such as "Hu Jiang away from the supply of Chi Xi, Xiu Qiu Lan that Pei", "hybrid pepper and bacteria Gui Xi, Qi Wei Fu Fu Hui", "Yu and so on, but also for the Yu-hui Xi, and Shen to the embrace of the "," to drink the magnolia of the mulberry Fall $\mathrm{Xi} \mathrm{Xi,} \mathrm{Xiqiu} \mathrm{Qiu} \mathrm{Ju} \mathrm{of} \mathrm{the} \mathrm{fall} \mathrm{of} \mathrm{the} \mathrm{British} \mathrm{"and} \mathrm{so} \mathrm{on,} \mathrm{it} \mathrm{is} \mathrm{too} \mathrm{numerous} \mathrm{to} \mathrm{mention.} \mathrm{It} \mathrm{should} \mathrm{be}$ said that in the use of natural style symbolic personality style, "Li Sao" is a precedent. In the imagination, reached the ultimate should also be the end of the driving fugue, which covers the "dragon", "Yao like" and "Tianhe" and many other magical images, and "start in Tianjin Come, Xi Yu Near the West "sentence, we can see the vertical and horizontal space span. In the vast expanse of space and a variety of magical images of the intertwined, an unpredictable, vertical and horizontal eighteen imagination of the world will be built on this. This is the "Lisao" idealistic style of a heavy expression, is the idealist style of the extension, but also the most obvious manifestation.

To some extent, this emphasis is implicit, because it is not like the extraordinary imagination can have a direct sense, in other words, it is idealistic style of a more profound embodiment. This is to be seen from the poet's own act and as an ideal pursuit of performance.

And go to "Lament", you can find a lot of such clues. Such as "Although it is not the people of this day comes, is willing to according to Peng Xian of the left", "also Yu Shen of the good Xi, although nine dead still regret it", "Ning death to exile Xi, I could not bear this state" V clear white to die straight, solid before the holy thick "," Although the body of the solution I have not changed Xi, Qi Yu heart can punish! "," The road is long Xi Xi Xi, I will be up and down and seek "and other words, it is this idealized protest of the direct show. This kind of struggle is mostly "somethin", "death", "death", "death" and other tragic way, but the focus is in the "Utah", that is, the poet to the power of the party and the world to compete, Destined to fail, but even knowing that in vain, but still so I, trying to use their own noble character to transform the "vulgar", even if their own destruction, its conduct should be maintained. So it is easy to see the ideal of this struggle.

\section{The Generation and Analysis of Realistic Style}

In the previous text, we have analyzed the weakness of Chu's rational culture from the perspective of the physical geography environment. Since the rational culture does not have any great influence in the culture of Chu, it does not dominate the realism. The Therefore, I think it is necessary to emphasize that the realism here is not the realism of the Chu culture, but the realism in the works of the Chu. This realism is not determined by the culture of Chu, but by the social background and political environment.

Qu Yuan's life is the era of the Warring States period of great changes in the history of the transition period, on the one hand the old slavery and ritual system, the rapid collapse of the river. On the other hand the emerging feudal system is in the ascendant and great struggle. Qin in the shift in the political environment to seize the opportunity to innovate and thus become a powerful country.

Qin's strong in the "Historical Records" has been seen in the "emperor eleven years, Jian will attack Zhao and, broken, pull NINETOWNS, eighteen years, Jian will attack Zhao, Yu Yu, then pull Zhao, Zhao Wang down, as far as possible for the county "(" Historical Records · white from Wang Jian Biography "). During this time of Chu, will also face the "best for the county" crisis. And facing the crisis of the situation, Chu Huai Wang in the country's governance but did not act as Chu Chuangxiong infighting, playing with the right way, so Qu Yuan described by the cloudy political environment on the formation of this.

"Solid and vulgar works of clever $\mathrm{Xi}$, it rules and change measures" is the time of Chu social background and a clear picture of the political environment.

But Qu Yuan's own positioning and living in the political environment is opposite, in the "Li Sao", open to clear that the mind said, "I have both inside the United States Xi, and heavy to repair. Hu Jiang away from the provision of Chi Xi, Xiu Qiu Lan that Pei "," do not give strong and abandoned dirty $\mathrm{Xi}$, why not change the degree of ", in the later also referred to" neither enough and for the United States and Xi, I will be from Peng Xian. " Whether it is Qu Yuan's own high-caliber or its "beauty" ideals, with the deadly dirty, undercurrent surging Chu's real political environment in 
conflict, and in the big environment, the principle of Qu Yuan became isolated hostile, so Qu Yuan was hit by it is not surprising. And as an isolated hostile, it should be said that Qu Yuan became the de facto "public criticism", by the political and psychological double blow.

In the case of political strikes, he mainly came to Qu Yuan's political opponents, such as Shangguan and his son, who were portrayed as "party", "maiden", "secular" and "present" In order to enhance and consolidate the status and vested interests in Chu, and must exclude Qu Yuan, so "the public jealousy of the Crescent" situation is gradually highlighted come out. Zilan and others in front of the king of the calumny of the already indecisive pregnant king more alienated Qu Yuan ("Huai Wang anger and Shu Ping"), then Qu Yuan was first relegated, and then exiled, which is Qu Yuan received Political blow.

Qu Yuan in the psychological impact of the blow, I think this blow has two main sources. One is Qu Yuan's own noble character and evil cloud of the social environment produced by the strong contrast and intense conflict caused by the loneliness of the pain, and the other is the political unsuccessful to Qu Yuan caused by the psychological blow.

The political blow to Qu Yuan is the source of the realism of his work, and the psychological blow to it is a direct clue to the realism of Qu Yuan's works.

This realism in the "Lisao" also reflected. As in the "Li Sao" has been mentioned in the "but this party does not understand $\mathrm{Xi}$, fear jealousy and the fold", "how can the body of the fear of Xi Xi, fear of Huang Yu's defeat", "I am not difficult to leave Xi, the spirit of the repair of the number of "are expressed in place, all because of the villain blinded monarch and make their own unsuccessful situation and psychological feelings, anger feeling overflowing words, depressed shape has vividly on paper. This is the direct expression of the poet in the political and psychological double hit after the natural reaction, so straightforward and targeted is a strong reflection of its realism. But in the "Li Sao" such as the imagination and symbolism-based full of idealistic color of the subject, so straightforward and obvious targeted statements of the weight is relatively light, so I think in the "Lament", The main way of his performance (imagination and symbol) is still idealistic style, and this realism is vivid, but we must fully affirm its existence.

\section{The Blending of Idealist Style and Realistic Style}

From this point of view, The Songs of Chu, only on the works of Qu Yuan, there is idealism and realism two different colors. But literary works are always complex, that is, the style of literary works are more or less complex, we can not be a certain class or a class of works are simply defined as a single style. Therefore, we say that The Songs of Chu itself is both idealistic and realistic. Through the previous analysis, we can see that in the idealistic style of the "LiSao" also "nine dead no regrets," the show, in a strong realism with the "Huai sand" also has a certain symbol of the composition exists, so it can support the proposed Song of the Song Dynasty has a double style characteristics. It is not difficult to see the idealist style and realism in the blending of the Songs. If only in a The Songs of Chu only idealistic style of dominance, it is easy to flow in the vague, if only to see the realism of which the works of literature will be greatly reduced. Therefore, we should say that we see today as the peak of poetry and the existence of The Songs of Chu, are the combination of the two products. It is the two styles of opposition and blending, only to create The Songs of Chu in the artistic and ideological on the very high achievements.

\section{The Chu to the Later Generations}

In addition it is worth mentioning that The Songs of Chu in the aesthetic tendencies and values on the later generations also had an indelible impact, more precisely, is a guide.

$\mathrm{Han} \mathrm{Fu}$ in the form and length are inherited the characteristics of The Songs of Chu, in the Han Dynasty appeared a large number of quasi-works, such as Jia Yi's "oath", the East Shuo "seven remonstrance", Huainan hills "recruiting" of the "sad life", Liu Xiang's "nine sighs", Wang Yi's "nine thinking" are the outstanding works of the proposed. They are not only in the form of "Li Sao" as the representative of the impact of the Songs of Chu, in the description of the way, the 
scenery also has obvious characteristics of the proposed, most also bearing the "LiSao" intention, express their own injustice and anger of the gas, Similarly, these quasi-works are mostly frustrated and made, at this point is also exactly the same with Chu.

Not only confined to Fu body, the aesthetic pursuit of The Songs of the Song has become an important paradigm of later poetry and prose aesthetic, that is, with a variety of scenery, and give its personality characteristics, the feelings of sincere and hearty, with the corresponding Is subtle timeless, of course, that is the "Book of Songs" of the force, and here for the time being not table. And the truth we speak is mainly represented by the boldly sent words, which is influenced by the lyrical way of The Songs of the Chu.

And in the value orientation, the impact of the Song Dynasty on the later generations is more obvious. Qu Yuan's works often show their feelings of family and country ("Huai sand", "orange song"), but also express the dark cast, worry about the peace of the people ("Li Sao"), covered ambitions and pursuits are later in the "Li Sao", Qu Yuan is a direct expression of the mind, "refers to nine days that is $\mathrm{Xi}$, husband but spiritual reason also", which has become the idea of loyalty to the earlier literary expression. Therefore, it can be said that the values and ideals pursued by The Songs of Chu have also played a great role in guiding and demonstrating the later literary creation and becoming the mainstream value of the later scholars.

\section{Conclusion}

At the beginning of our paper, we put forward the "Songs of the South both the idealist style and the realism of color," the core proposition, and then separately explore the idealist style and the formation and performance of realism. And then explain the two blend of each other on the great achievements of The Songs of Chu. Finally, it also expounds the guiding role of The Songs of Chu in the art and thought to the later literature, and further affirmed the foundation and pioneering position of Chu.

The Songs of Chu is one of the two great estrus of Chinese poetry. For the study of The Songs of Chu, the predecessors have prepared, and I have also made some opinions in this article. If there are one a few commendable points, it will be my honor, and there are also some imperfect opinions, if so, I hope that the leaders could guide us.

\section{References}

[1] Luo Zongqiang. "Chinese ancient literature works selection" pre-Qin and Han Dynasties volumes "Higher Education Press 2004 edition

[2] Sima Qian. "Historical Records · Bai Qi Wang Jian Biography" Sanqin Publishing House 2008 edition

[3] Sima Qian. "Historical Records · Qu Yuan Jia Sheng Biography" Sanqin Publishing House 2008 edition

[4] Mei Qionglin. "on the attributive function of $\mathrm{Qu} \mathrm{Fu}$ literature and its potential cultural background." Jianghan Forum, 1998 (3): 45-48

[5] Xiong Liangzhi. "the value of the study of the Songs of Chu". Journal of Sichuan Normal University (Social Science Edition), 1999 (4): 49-57

[6] Li Jinkun. "Central Plains culture influence Chu culture six" Journal of Jiangsu Education College, 2006 (2): 47-50

[7] Lin Jiali. "The Songs of Chu" Zhonghua Book Company 2016 edition 